\title{
The task of multi-criteria optimization of metal frame structures
}

\author{
Vadim Alpatov ${ }^{1, *}$ \\ ${ }^{1}$ Samara State Technical University, Institute of Architecture and Civil Engineering, \\ 194, Molodogvardeyskaya St., 443001, Samara, Russia
}

\begin{abstract}
Optimal design of a frame structure with a specified geometric scheme consists in finding control parameters that provide the highest or lowest value of composite functions which present some quality criteria. Searching for optimal parameters is related to a number of design and calculation constraints. When it is necessary to vary a geometrical scheme, node coordinates are also considered as unknown varied parameters that affect the quality criteria. When designing frames with a specified scheme, the volume of material is typically the primary criterion for solving an optimization task and is written as a function of control parameters and state settings. In problem specification it is also important to reduce the deformation of the system. This is accomplished by introducing an additional criterion -maximum moments of inertia of the sections of the system. There is a two-phase design and calculation model existing in design practice now. In the first stage, the work is based on the experience or existing prototype. On their basis stiffness of the bars is assigned, and then a load vector is calculated. In the second stage, the sections are chosen according to known forces.
\end{abstract}

\section{Introduction}

Optimal design of a frame structure with a specified geometric scheme consists in finding control parameters that provide the highest or lowest value of composite functions which present some quality criteria. Searching for optimal parameters is related to a number of design and calculation constraints [1-3].

The dimensions of cross sections of metal elements (width, thickness, and surface of rolling parts) are the basic parameters from which the system properties change. These settings are called controlling variables.

When it is necessary to vary a geometrical scheme, node coordinates are also considered as unknown varied parameters that affect the quality criteria. The search for an optimum scheme of a structure or a construction should be based on a algorithmic comparison of quality criteria for individual cases $[4,5]$.

*Corresponding author: avu75@mail.ru 


\section{Materials and methods}

As any frame structure consists of many elements, the vector of the system control variables itself consists of vectors of parameters of each of its constituent bars, that is:

$$
Z=\left(Z_{1}, Z_{2}, \ldots, Z_{m}\right),
$$

where $m$ - is the number of bars in the system.

Each embedded vector $Z_{i}(i=1,2, \ldots, m)$ looks like this:

$$
Z_{i}=\left(Z_{1}, Z_{2}, \ldots, Z_{d i}\right)
$$

where $d_{i}$ is the number of control variables in the bar number $i$.

When choosing $Z_{k}$, the following condition must be observed

$$
Z_{k} \in D_{k}
$$

Here $D_{k}$ is a limited set, where $k=1,2, \ldots, m_{1} ; m_{1}$ - the total amount of typycal sizes of elements.

Let us specify control parameters by using double indexing, as $Z_{j k}(j=1,2, \ldots, d)$, then the vector of the control variables is written as:

$$
Z=Z\left(Z_{j k}\right), Z_{j k} \in D_{k}, Z_{j k} \geq 0 \text {. }
$$

To develop a mathematical model for finding an optimal solution for frame structures, we have to consider parameters defining geometry of the system and impact of external environment on the system in question (topology of the construction, node coordinates, loads, and other). At the first stage, we accept these parameters as fixed. Let's call them status parameters. A vector of status parameters of the frame structure includes state vectors of individual elements:

$$
B=\left(B_{1}, B_{2}, \ldots, B_{p}, \ldots, B_{m_{3}}\right) .
$$

Here $B_{p}=\left(b_{1 p}, b_{2 p}, \ldots b_{z p}\right)$ is the state vector corresponding to the load $p ; p=1,2, \ldots$, $m_{3}$.

When designing frame structures with a specified geometric scheme, the volume of material is typically the primary criterion for solving an optimization task and is written as a function of control parameters and status parameters.

$$
V=V(Z, B)=\min .
$$

When specifying an optimization task, it is important to take into account the requirement to limits of the system deformation . It can be achieved, for example, by introducing the second criterion = maximum moments of inertia of sections of the system:

$$
I=\left[I_{1}\left(Z_{1}\right), I_{2}\left(Z_{2}\right), \ldots, I_{z}\left(Z_{d}\right)\right]=\max .
$$

Some properties connected with energy condition of the system can be shown as a vector of minimized composite functions: 


$$
\phi=\left[\phi\left(Z, B_{1}\right), \phi\left(Z, B_{2}\right), \ldots \phi_{m 3}\left(Z, B_{3}\right)\right]=\min .
$$

The same properties can be written in the form of constraints-equalities, such as an equation of the force method or the method of movement.

Restrictions should be placed on construction and design characteristics of elements of the system. Such restrictions are presented in the form of approximate equalities:

$$
G(Z, B) \geq 0,
$$

where: $G(Z, B)=\left[G_{1}(Z, B), G_{2}(Z, B), \ldots, G_{m 2}(Z, B)\right] ; m_{2}$ - the number of constraint vectors.

There is a two-phase design and calculation model existing in design practice of building structures now. In the first stage, the work is based on the experience or existing prototype. On their basis stiffness of the bars is assigned, and then a load vector is calculated.

$$
X=\left(X_{1}, X_{2}, \ldots, X_{d}\right)
$$

Here $X_{i}=X_{i 1}, X_{i 2}, \ldots, X_{i m 3}$ - the forces in bar $i$.

In the second stage, the sections (their parametres) $Z$ are chosen according to known forces $X$.

This two-stage design model can be used for optimal design of frame structures with specified geometry. If it is necessary to find the system's geometric shape, the task becomes a three-stage one with an additional set of varied parameters: geometric parametres of the system. In this case, the solution of the generalized optimization task should be organized in stages, based on its decomposition. It is proved that the convergence of this process to the global minimum will be ensured if the main criterion and the limitations expressed in $X_{i}$ are bumped [6].

At the level of one bar of the system, the search for its optimum cross-section (see Figure 1) can be reduced to a variation and selection from the gauge material of discrete characteristics of the compound section (flange, wall, edge, etc.) satisfying several quality criteria, design standards requirements, and manufacturing and mounting conditions. The algorithm described can be applied both to the private task of finding optimal section parameters of a certain type, and of solving the problem of finding the optimal section shape, steel grade, or section dimention.
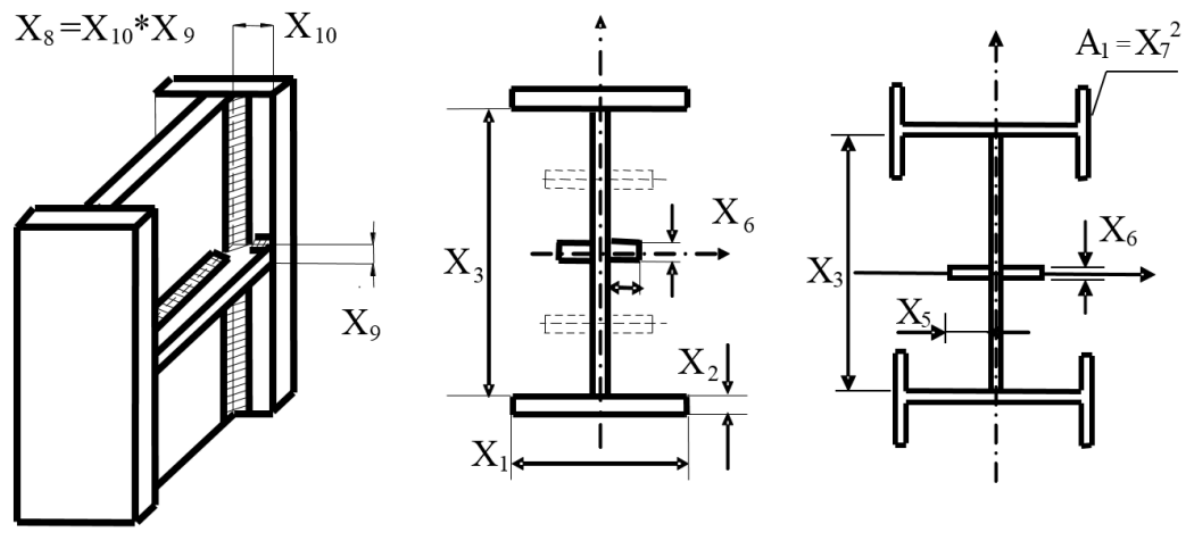

Fig. 1. Cross-sections diagram. 
It is known that there are many best and closest to the best solutions to the optimum. Finding multitudes that are called pareto-optimal in the tasks of multi-criteria analysis is the purpose of the this work.

Volume, labour input, and rigidity criteria can be presented in general terms by expressing them through the system's accepted parameters, that is, by formulating three target functions:

$$
\begin{gathered}
V=\sum_{i=1}^{m_{1}} \sum_{j=1}^{m_{2}} a_{i j} \cdot X_{i} \cdot X_{j} ; \mid X_{i} \in B_{1}\left(B_{3}\right) ; X_{j} \in B_{2}\left(B_{3}\right) \\
T=K \cdot \sqrt{\left(\sum_{i=1}^{m_{1}} \sum_{j=1}^{m_{2}} a_{i j} \cdot X_{i} \cdot X_{j}\right) \cdot \gamma \cdot n} \\
I=\sum_{i=1}^{m_{1}} \sum_{j=1}^{m_{2}} a_{i j} \cdot X_{i} \cdot X_{j} \cdot\left(k_{i j} \cdot X_{j}^{2}+b_{i j}\right),
\end{gathered}
$$

where $k_{i j}=n_{i} / 12, b_{i j}=n_{i} \delta_{i}^{2}$ ( $n_{i}-$ - the number of elements in the section).

Equations (11)-(13) are hypersurfaces in the sphere of accepted parameters $X_{i}$ and $X_{j}$. Hypersurfaces $V, T, J$ have a number of important features:

1. they are are non-convex, so there is no limit to the minimum (except for a trivial one) without additional restrictions;

2. they ascend in the increase $X_{i(j)}$ (when $X_{i(j)}>0$ );

3. their minimum lies in the area adjacent to the boundary of the restrictions;

4. their optimum can be multi-valued when there are several combinations of pairs $X_{i}, X_{j}$ or the same volume or there are slightly different (from the optimum) solutions (quasi optimal).

The view of surfaces $V, T, I$ when $X_{i}>0 ; X_{j}>0$ is shown on Fig 2.

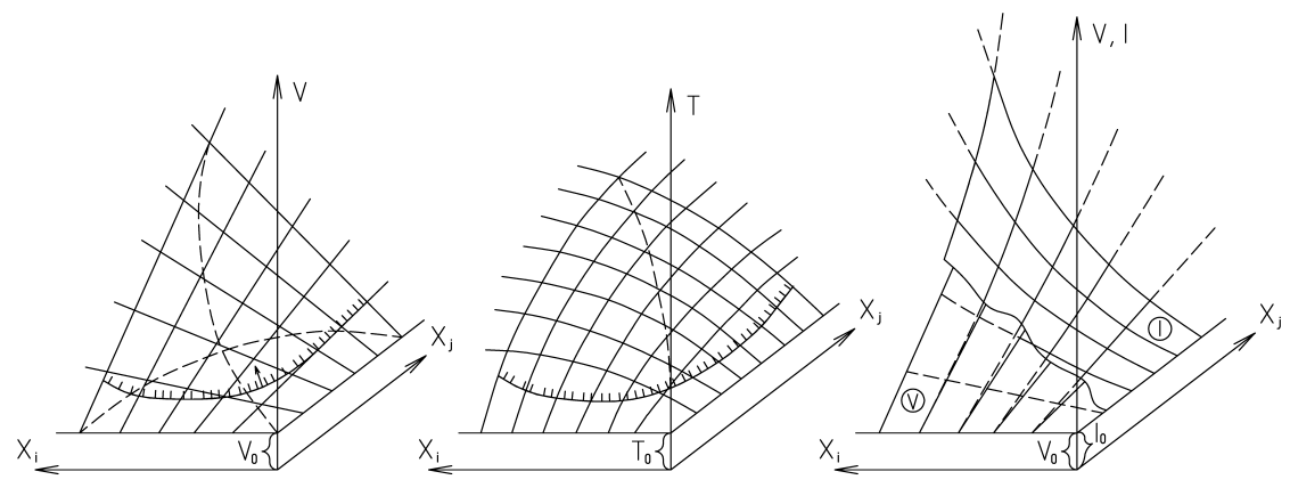

Fig. 2. Hypersurfaces of criteria $V, T, I$.

The limitations placed on target functions and system parameters can be divided into two types: constructive limitations and design limitations.

Constructive limitations are related to the limits of parameter ratios introduced by construction regulations. They can be presented as:

$$
f_{k}\left(X_{i}, X_{j}\right)-C_{k} \geq 0
$$

Here $k$ is the restriction number $C_{k}=$ const, $D_{1} \leq X_{i} \leq D_{2} ; D_{3} \leq X_{j} \leq D_{4} ; X_{i}>0$; $X_{j}>0$. 
Limitations on strength, general and local sustainability are as fpllows:

$$
f_{k}\left(X_{i}, X_{j}\right)-g_{k}\left(X_{i}, X_{j}\right) \geq 0
$$

When optimizing according to multiple criteria, a method of succesive concessions can be applied [7]. In this case, the first step is to find a minimum value of the leading criterion $V\left(X_{i}, X_{j}\right)=\min$. Besides, $X_{i} \in G_{1}, X_{j} \in G_{2}, G_{1} \subset B_{1} ; G_{2} \subset B_{2}$. Multiples $G_{1}$ and $G_{2}$ are formed from $B_{1}$ and $B_{2}$ under limitations. In the second stage, criteria limitations are formulated.

$$
\begin{aligned}
& \min V \leq V \leq V^{*} \mid V^{*}=\left[\min V+\varepsilon_{1}(\min V)\right], \\
& \min T \leq T \leq T^{*} \mid T^{*}=\left[\min T+\varepsilon_{2}(\min T)\right] \\
& \max I \geq I \geq I^{*} \mid I^{*}=\left[\max I-\varepsilon_{3}(\max I)\right] .
\end{aligned}
$$

Here, $\varepsilon_{1}(\min V), \varepsilon_{2}(T \min ), \varepsilon_{3}(\max I)-$ the values of concession", by volume, labour input and rigidity, respectively.

The selection of optimum sets is based on the solution of two minor tasks:

1. Defining the standard $\left(V^{o n t} ; T^{o n t} ; I^{o n t}\right)$.

2. Formation of many permissible (pareto-optimal) solutions.

The search for the optimum consists of the following operations:

1. Fixation $X_{1}, X_{2}, \ldots, X_{n-1}, X_{n} \in B_{1}, B_{2}$;

2. Verifying the ability to exhaust the set step of $B_{1}, B_{2}$;

3. Variation $X_{i}, X_{j}$ in a manner that provides $I \rightarrow \max$ and defines $\min V, \min T$;

4. Finding rigidity appropriate to its critical limitation.

If the solution is found for the first time, it is taken for a standard $V_{1}^{0}=V_{1}, T_{1}^{0}=T_{1}, I_{1}^{0}=I_{1}$. The subsequent variation analyzes the optimum by comparing $V_{n}$ with $V_{n-1}^{0}$, where $n$ is the step number. There are three possible options:

1. If $V_{n} \leq V_{n-1}^{0}\left(1+\varepsilon_{1}\right)$ and $I_{n}>I_{n-1}^{0}$, then the decision $n$ is optimum and accepted as a standard;

2. If $V_{n-1}^{0} \leq V_{n} \leq V_{n-1}^{0}\left(1+\varepsilon_{1}\right)$, a $I_{n-1}\left(1-\varepsilon_{2}\right) \leq I_{n} \leq I_{n-1}^{0}$, then the standard for comparison is still $V_{n-1}^{o}$ and $I_{n-1}^{o}$, though $V_{n}$ and $I_{n}$ are remembered as valid (multitude $D)$;

3. If $V_{n}>V_{n-1}^{\mathrm{o}}\left(1+\varepsilon_{1}\right)$, the decision is discarded even before limitations are checked.

Criteria limitations in the optimization process are specifies when the standard $V^{\mathrm{o}}, T^{\mathrm{o}}, I^{\mathrm{o}}$ is specified. Therefore, after $D$ is found, a final sorting is performed and a multitude of effective (Pareto) points is allocated. Then the search for optimal sections for the specified parameters is over.

In a discrete task, there are many equivalent options for optimal variants and variants closest to optimal. Therefore, the optimum scope will be fully defined only after all parameters have been traversed. At the same time, there are areas where it is meaningless to traverse parameter combinations because they do not provide any structural, technological or computational limitations. That is why it makes sense to use a method direct search of 
the values of discrete parameters when performing a series of "cutting-off multitudes" techniques.

\section{Results}

The above method of solving a multi-criteria task through the sequential concessions is implemented in a computing complex developed in Samara state university of architecture and civil engineering. The complex is designed to optimize the selection of steel structure sections with different section topologies, steel marks and dimensions.

For space-framed constructions, questions of optimal design are most actual in relation to their weights or labour input indicators. For such structures, because of their constructive features, the contradiction between materials consumption and labour input is most evident. Traditionally for such constructs (in the case of serial and generic structural slabs), the design priority was to reduce labour intensity by simplifying the design, including joint connection. This was often done at the expense of their materials consumption. Therefore, because of the large number of elements, firstly, and because of the strict unification of elements of structural constructions, secondly, they are characterized by increased material production discharge. Much of the cost of these designs is offset by a decrease in labour input in manufacturing, transport and assembling. However, today there is no convincing justification for the advantages of such a strict unification for any known spatial-frame structures. An economic justification of this type can only be given only when there is a task of optimizing with a clear mathematical formulation of the problem is solved. It is obvious, however, that this problem should be raised either as a multi-criteriatask of parametric optimization, or in an even broader setting, as a function of synthesizing a structure. It follows from the above that at least two criteria, mass and labour input, should be introduced in the formulation of such tasks. When designing real-world objects, there is typically a need to take into account many additional factors (such as rigidity, etc.) that can be entered as limitations or as quality criteria in a structural optimization task. In the latter case, there is an optimization task with more than two criteria.

The author of this paper has solved a number of tasks for optimizing space-framed constructions [8-10], [20]. These tasks were formulated as a search for optimal geometry of structural constructions. Because of the complexity of the mathematical model of the task, it was devided into two subtasks, that is, the decomposition was performed. Decomposition of the tasks was accomplished by using the hierarchy of parameters, limitations, and target functions for each subtask. Each subtask there was only a stage of optimization for the main task, but at the same time was an independent complete optimization task.

Splitting into two subtasks is due to the fact that an iterative recalculation method was adopted to solve the main task. The search for a solution requires two stages of optimization: finding optimal geometry of the structural construction and finding optimum distribution of the material in the structural construction with the unchanged geometry. The two-stage devision is natural in terms of definition with variable and non-variable parameters at each stage of the design, the form of a mathematical record of the target functions and the limitations expressed through the accepted parameters. Dividing the main task into two phases is natural and in terms of classifying optimization tasks [7], [8], [14$16]$.

\section{Discussion}

In general, the task of a high hierarchy is to find optimal geometry of a structural construction. It is formulated as follows: 
Let us assume, that there is a structural construction with topology and geometry in a certain plan (a span in one direction, a span in the other direction). Its support conditions, materials of the elements, and the structure load diagram are known. We need to define an optimal geometric shape of the structural construction.

A low-hierarchy task is to find the optimum distribution of material in a structural construction with unchangeable geometry. It is formulated as follows:

Let us assume there is a structural construction with a specific topology and geometry. It means we know a span in one direction, a span in the other direction, coordinates of all structure nodes. The conditions of the support, material of the elements and the structure load diagram (see Figure 3) are also defined. We need to define optimum distribution of the material in a structural construction when the mass of the structure is minimal.

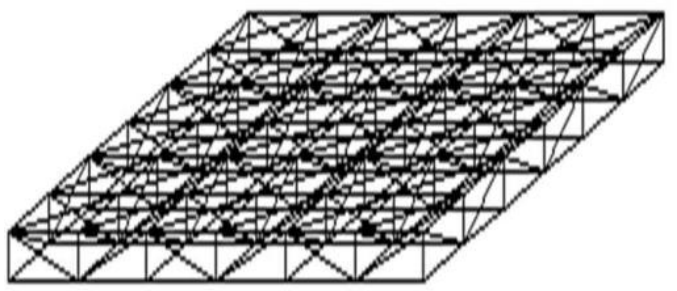

Fig. 3. Structural plate.

For the first task, a solution algorithm was implemented. It presented an iterative process to find the minimum mass based on the random search method. The task was formulated and solved as a single-criterion (with mass as a criterion) task.

For the second task, the methodology described above, the algorithm and the programme complex on their basis [15], [17] were applied. This task was solved as a twocriteria task, with criteria bing volume and rigidity.

The implementation of the mathematical model described above resulted in the creation of a software complex for calculation and optimization of structural constructions. The complex is called "Search for the optimal form of structural constructions." The complex operates in a computer-to-people dialogue mode: static analysis of structural constructions; recalculation of bar sections; optimization calculations of proportioning elements sections based on the discrete size of the rolling profiles according to minimum volume and maximum rigidity criteria; search for optimum structural structure geometry on the minimum mass criterion [18], [19].

\section{Conclusions}

Figure 4 shows some of spatial constructions that resulted from finding the optimal geometric shape of these structures when using the introduced software complex.
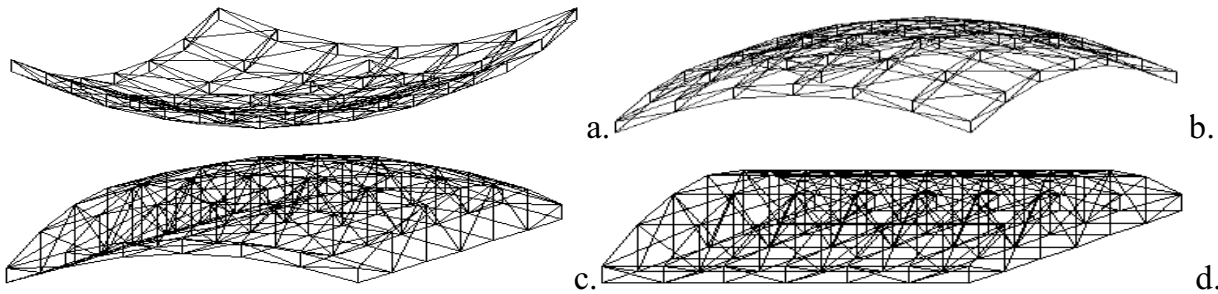

Fig. 4. Spatial structures obtained when searching for optimal geometry. 


\section{References}

1. I.S. Kholopov, Vestnik of Research Science Center "Building Construction" 2, 114125 (2010)

2. I.S. Kholopov, Industrial and civil engineering 8, 13-17 (2010)

3. I.S. Kholopov, Metal structures 4, 285-297 (2008)

4. A.V. Ginzburg, A.A. Vasilkin, Vestnik MGSU 6, 52-62 (2014)

5. M.R. Garifullin, S.A. Semenov, S.V. Belyaeva and others, Construction of unique buildings and structures 2 (17), 107-124 (2014)

6. L.S. Lasdon, Optimizing large systems (Moscow, Science, 1975)

7. I.M. Rabinovich, On the theory of static undefined trusses (Moscow, Transzheldorizdat, 1933)

8. V.Yu. Alpatov, Thesis for PhD in Technical Sciences (2002)

9. V.Yu. Alpatov, Efficient building structures: Theory and practice, 199-206 (2002)

10. V.Yu. Alpatov, I.S. Kholopov, Actual problems of improvement and development of metallic, wooden and plastic structures in construction and transport, 193-198 (2002)

11. V.Yu. Alpatov, Actual problems in construction and architecture. Education. Science. Practice, 476-477 (2007)

12. A.O. Lukin, V.Yu. Alpatov, D.D. Chernyshev, Urban Construction and Architecture 2(23), 4-9 (2016)

13. A.I. Vinogradov, Papers of Kharkov Institute of Railway Engineering 25, 30-36 (1955)

14. A.I. Vinogradov, Study on construction theory (Moscow, Gosstrojizdat, 1965)

15. I.S. Kholopov, Thesis for Ph.D. in Technical Sciences (1992)

16. I.B. Lazarev, Basics of optimal design. Tasks and methods (Novosibirsk, SGAPS, 1995)

17. I.S. Kholopov, A.V. Solovyev, A.V. Simbirev, The 59th Scientific and Technical Conference abstracts, 44 (2002)

18. V.Yu. Alpatov, The 56th scientific and Technical Conference abstracts, 277-278 (1999)

19. V.Yu. Alpatov, Postgraduate Bullitin of Samara region 2, 83-86 (2001)

20. V.Yu. Alpatov, A.O, Lukin, A.A. Sakharov, D.I. Zhuchenko, Urban Construction and Architecture 4(25), 19-22 (2016) 